\title{
CALCULATION OF THE EFFECTIVE ABSORPTION COEFFICIENT IN MULTI-PHASE POWDERS
}

\author{
D. REEFMAN \\ Philips Research Laboratories, Prof. Holstlaan 4, 5656 AA Eindhoven, \\ The Netherlands
}

(Received 14 February 1995)

\begin{abstract}
A calculation of the effective absorption coefficient $\mu^{\text {eff }}$ in multi-phase powders is presented. Characteristic in the calculation is, that the distribution function of the grains is similar to the correlation function for fluids. It is shown, that for average grain diameters comparable to the inverse of the absorption coefficients, the commonly used formulae for $\mu^{\text {eff }}$ (either a linear combination of the constituting $\mu$ 's or simply $\mu$ for a single component) do not hold.

The analytical expressions derived for $\mu^{\text {eff }}$ are compared with numerical simulations, which show that,

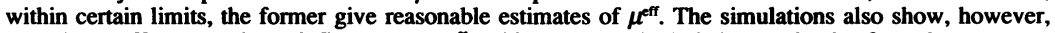
that shape effects can have influence on $\mu^{\text {fff }}$, which are not included (yet) in the formulae.

Also shown is that surface roughness can give effects comparable to those of microabsorption, but can be much more sensitive to particle statistics.
\end{abstract}

KEY WORDS: Micro-absorption, intensity, surface-roughness preferred-orientation.

\section{INTRODUCTION}

In many X-Ray applications, e.g. quantitative phase analysis (QPA), Rietveld refinement, or ab-initio structure determination, correct values for the reflected intensities are of utmost importance. These procedures are therefore highly apt to errors, arising from spurious effects causing changes in intensities.

Errors due to texture have been addressed extensively by e.g. Bunge et al. (1992) and by Ahtee (1989), and at least theoretically the problem seems to have been solved to a great extent.

Depending on the radiation wavelenght and the sample properties, microabsorption effects can be of importance. For $\mathrm{Cu}$ radiation and a $\mathrm{Si}$ powder sample (with absorption coefficient $\mu=140 \mathrm{~cm}^{-1}$ ), the often used concentration weighted absorption coefficient for experimental absorption correction only holds for grain diameters smaller than roughly $10 \mu \mathrm{m}$. The other limit, where the unaffected $\mu$ is used for the correction, holds for grain diameters larger than $500 \mu \mathrm{m}$. For materials with larger $\mu$ 's, e.g. transition metals with $\mu \sim 1000 \mathrm{~cm}^{-1}$, the lower and upper limits of the grain diameters change to roughly $2 \mu \mathrm{m}$ and $50 \mu \mathrm{m}$. Obviously, the experimentally realized situations are often outside these validity regions.

The effects of microabsorption and surface roughness have been recognized and addressed by several people. The importance of the absorption contrast has been pointed out already by Brindley (1945). The first major attempt to describe, at least semi empirically, the effects of the non-homogeneity were made by Suortti (1972), who also 
separated surface roughness effects from bulk contributions. A first attempt to solve the problem numerically has been made by Otto (1984). Due to the fact, however, that a simple two dimensional model was used, with uniform grain diameters, the results are not very general. Hermann and Ermrich (1992) presented an analytical treatment. However, the approach they used allowed for overlapping grains in the specimen. Moreover, it is only valid in multi-component mixtures where all components except one have absorption coefficients $\mu$ of about equal magnitude.

In the present paper, we address the problem of microabsorption in detail. In sec. 2.1 , an analytical model is presented based on correlation functions as they have been calculated for hard sphere fluids. In section 2.2, a more general numerical approach is described. The results from both methods are displayed and compared in section

3. Finally, in section 4, conclusions and an outlook are presented.

\section{THE MODEL}

\subsection{Analytical approach}

The first question to address is how to define an 'effective absorption coefficient', $\mu$ eff. For a homogeneous powder with filling fraction $n$, we have the following relationship for the reflected intensity $I$, if the materials absorption coefficient is $\mu$ :

$I=\frac{I_{0}(\theta) n}{\sin \theta} \frac{1}{A} \int_{A} d x d y \int_{-\infty}^{0} d z R(\vec{r}, \theta) \mathrm{e}^{-2 \mu z / \sin \theta} \stackrel{\mathrm{R}=1}{=} n \frac{I_{0}}{2 \mu}$

where $\vec{r}=(z, y, z) ; z$ is the depth where the beam with incidence angle $\theta$ (symmetric geometry) is reflected; $I_{0}(\theta)$ is the intensity of the incident beam; $A$ is the irridiated area. Note that geometry influences on the intensity are in $I_{0}(\theta) . R(\vec{r}, \theta)$ is a reflectivity, which usually is assigned the numerical value of 1, leading to the equality in Eq. (1). Thus, generalizing for a heterogeneous powder, this gives a definition of the effective absorption coefficient $\mu_{t}^{\text {eff }}$ for phase $i$ :

$$
\mu_{t}^{\text {eff }}=n_{i} \frac{I_{0}}{2 I^{(i)}}
$$

In the limit of a homogeneous powder, $\mu^{\text {eff }}$ equals $\langle\mu\rangle=\Sigma_{i} n_{i} \mu_{i}$, where $\mu_{i}$ is the linear absorption coefficient of phase $i$. It is important to note that the presence of an absorption coefficient (and a reflectivity coefficient) in Eq. (1) simply is an attempt to correct the deficiences of kinematical diffraction theory. This is especially clear from Eq. (2), where it can be seen that for small absorption coefficients the calculated reflected intensity may even be larger than the incident intensity. A rigorous approach should therefore include extinction effects.

We now have to develop an expression for the total reflected intensity in a heterogeneous powder in terms of the concentration and particle sizes. The model we adopt to describe the heterogeneous powder, is depicted in Figure 1. Each component $i$ in the multi-phase system is characterised by a fraction $n_{i}, 0 \leq n_{i}<1$, and a (number) average particle diameter $\langle d\rangle$. In order to calculate the total reflected intensity, we have to take into account the fact that absorption only takes place in those parts of the traversed path that lie within a particle. Here, the approximation is made that the non- 


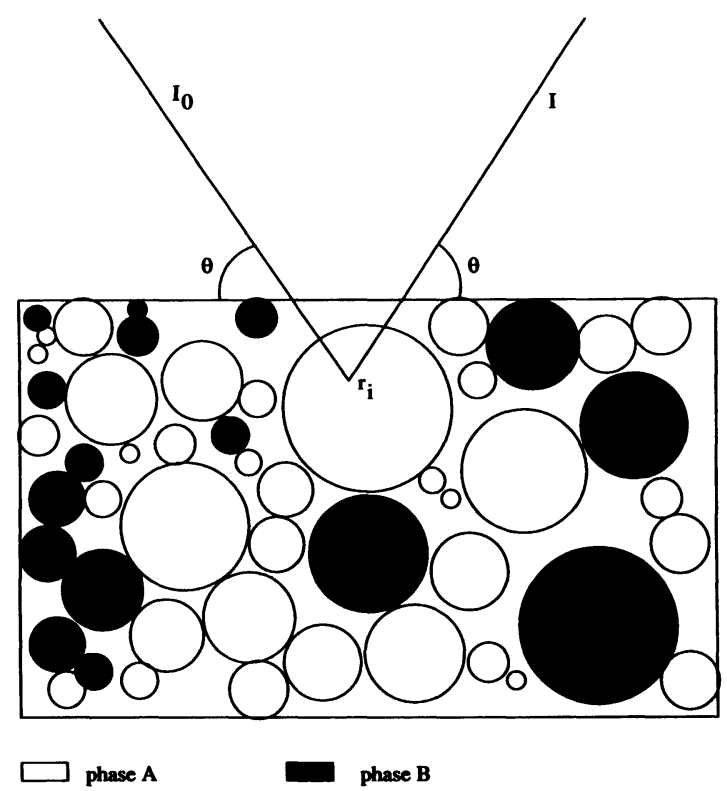

Figure 1 Model used to idealize a real sample. For the analytical calculations, only sperical shaped particles are allowed, for the numerical calculations ellipsoids.

homogeneity of the powder can be described statistically. A related question arises in dense fluid mechanics, where particle-particle correlations are important. There, a density-density correlation function is defined as: $g(r)=\langle\rho(0) \rho(r)\rangle /\langle\rho\rangle^{2}$ where $\rho$ is the average fluid density, and $\rho(r)$ the average fluid density at a distance $r$ from the (arbitrary) origin. In the present study, the correlation function for a hard-sphere fluid (Reichl 1980) is used as a basis for this purpose:

$$
g(r)=1-\frac{1}{r^{\prime}} J_{1}\left(2 r^{\prime}\right) ; \quad r^{\prime}=\frac{\mathrm{r}}{d^{\prime}}
$$

Here $J_{1}$ is a Bessel function of the first order, $d^{\prime}$ is the sphere diameter. If we allow for a gaussian distribution of diameters, and assume that the diameters for the particles belonging to different phases are approximately the same, the following probability distribution functions describe the inhomogeneiety of the powder:

$$
\begin{aligned}
& G_{i i}(r)=n_{i} \int_{0}^{\infty} d p e^{((r-p)(d)) 2}[\delta(p)+g(p)] \\
& G_{i j}(r)=n_{j} \int_{0}^{\infty} d p e^{((r-p))(d)) 2} g(p)
\end{aligned}
$$


where $\delta$ is the Dirac delta function, representing the correlation of the particle with itself. The first equation in Eq. (4) gives the probability that, given the fact that the reflection is in a grain of phase $i$ (i.e., $r=0$ is assumed to be the center of that grain), the phase at a distance $r$ is phase $i$. The second equation in Eq. (4) gives the probability that the phase at distance $r$ is phase $j$.

The effective path length $L^{\text {eff }}$, over which absorption of an X-ray reflected in phase $i$ at depth $z$ occurs due to phase $j$, can now be written as:

$$
L_{i j}^{e f f}=2 \int_{0}^{z / \sin \theta} d l G_{i j}(l)
$$

The total reflected intensity of a reflection of phase $i$ is now expressed as:

$$
I_{i}=I_{0} \int_{-\infty}^{0} \frac{d z}{\sin \theta} e^{\left[\mu_{t} L_{\text {fff }}^{\text {ff }}+\Sigma_{j \neq i} \mu L_{i f}^{\text {eff }}\right]}
$$

From this equation, the expression for $\mu^{\text {eff }}$ follows immediately from Eq. 2 .

\subsection{Numerical approach}

The basic principle in the numerical approach is not different from that for the analytical approach. Some restrictions, however, are relieved, and much more freedom is present in the choice of the microstructure of the powder.

In this approach, a particle is modeled as an ellipsoid, which may have both randomly chosen dimensions and directions (i.e., 6 variable parameters). The direction may be chosen from a non-uniform distribution, thus introducing a certain texture. A 3D box is than created, and filled with these particles until a certain filling degree is reached.

After this filling procedure, several quantities can be calculated, such as $G_{i j}(r)$ and $\mu_{i}^{\text {eff }}$. Though, after determination of $G_{i j}(r), \mu_{i}^{\text {eff }}$ can be determined using Eq. (6), we chose to calculate $\mu_{i}^{\text {eff }}$ directly because this is the more efficient way. The reflected intensity was calculated according to

$$
I_{i}=\frac{I_{0}}{V} \int_{V} d \vec{r}^{\prime \prime} f_{i}\left(\vec{r}^{\prime}\right) \int_{L(\vec{r})} d \vec{r} e^{-2 \mu(\vec{r}) / / \sin \theta} \sin \theta^{-1} \quad f_{i}(\vec{r})=\left\{\begin{array}{l}
1 \text { if } \vec{r} \text { in phase } i \\
0 \text { otherwise }
\end{array}\right.
$$

from which $\mu^{\text {eff }}$ can be determined.

The integral (7) was evaluated with a Monte Carlo method, chosing an exponential distribution for the random generation of the $z$ component of the positions $\vec{r}_{i}$ in the integration process in order to speed up the convergence:

$$
z=-\ln \left(z^{\prime} \sin \theta / \mu^{\mathrm{ary}}\right) \sin \theta / \mu^{\mathrm{try}} ; \quad \mu^{\mathrm{ry}}=\sum_{i} n_{j} \mu_{i}
$$

Here, $z^{\prime}$ is chosen from a uniform distribution $[0,1]$. Typically, in a run up to $10^{4}$ particles were used, and in the MC integration more than 80000 steps were used. Also, the 'illuminated area' was kept constant, in order to avoid artificial $\theta$ dependencies of the reflected intensities. In order to avoid effects due to low packing fractions, which complicate the interpretation, packing fractions have been chosen to be roughly 0.92 . Usually, real packing densities are lower (of the order of 0.6-0.8). 


\section{RESULTS AND DISCUSSION}

In this section, the units of $\mu$ have been chosen as $\langle d\rangle^{-1}$. As $\langle d\rangle$ has been taken to equal 1, the values for $\mu$ used below are simply numbers. In what follows, calculations have been performed for a dense two-component system, except in the section on surface roughness (where only a single component is used).

\subsection{Sperical particles}

In order to have an idea for the validity of both the analytical and numerical approach, we first present in Figure 2 some results for the case where $\langle\mu\rangle<0.3$. We expect, that in the case where $\langle\mu\rangle \ll 1$, the standard expression for $\mu^{\text {eff }}$ should hold:

$$
\mu_{\text {eff }}=n_{\mathrm{A}} \mu_{\mathrm{A}}+n_{\mathrm{B}} \mu_{\mathrm{B}}
$$

Indeed we see that, certainly in the range where $\mu_{A} / \mu_{B} \ll 1$, the expected simple relation (9) (dashed lines in Figure 2) between $\mu^{\text {eff }}$ and the concentrations holds rather well. Only for the higher $\mu_{A} / \mu_{B}$ ratios deviations occur, which is expected. Over the whole region however, the analytical and numerical determined absorption coefficients are in good agreement.

In going to large values of $\mu_{A}$, another limit should be approached, namely that where:

$$
\mu_{i}^{\mathrm{eff}}=\mu_{i}
$$

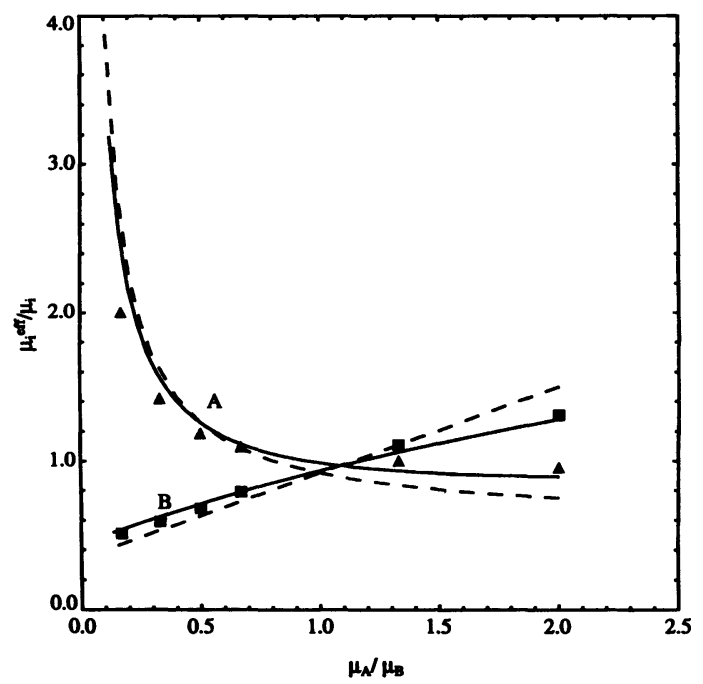

Figure 2 Results according to Eq. (6) for $\mu_{A}^{\text {eff }} / \mu_{A}$ (A) and $\mu_{B}^{\text {eff }} / \mu_{B}$ (B) as a function of $\mu_{A} / \mu_{B} ; \mu_{B}=$ 0.3 (solid lines). The dashed lines represent the same according to Eq. (9). The results from the simulation are marked by triangles $\left(\mu_{A}^{\text {eff }} / \mu_{A}\right)$ and by squares $\left(\mu_{B}^{\text {eff }} / \mu_{B}\right)$. The packing density is 0.92 , $n_{A}=0.58, n_{B}=0.34$. 
Strictly, this relation is valid only in a sample with a filling factor equal to 1 without surface roughness. Nevertheless, we see that both in the analytical and numerical evaluations of $\mu^{\text {eff }} \mu_{A}^{\text {eff }} / \mu_{A}$ approaches 1. For component $B$ we still are far from this limit.

In Figure 3, some results for higher $\mu$ values $(\langle\mu\rangle \sim 4)$ are depicted. Though qualitatively the analytical results for high $\mu$ do not differ from those for low $\mu$, the quantitative differences are large. As expected, $\mu_{B}^{\text {eff }} / \mu_{B}$ is almost insensitive to variations in $\mu_{A}$, and equals 1 (cf. Eq. 10). On the contrary, $\mu_{A}^{\text {eff }} / \mu_{A}$ is still sensitive to variations in $\mu_{A}$ at low $\mu_{A}$, though this effect is not as strong as for low $\mu$. The numerical results in contrast, show a behaviour which is even qualitatively different. The dependence of $\mu_{B}^{\text {eff }}$ on $\mu_{A}$ is far less smooth than in Figure 2 . For very low $\mu_{A}, \mu_{B}^{\text {eff }}$ also is significantly lower than $\mu_{B}$. This in fact is reasonable as long as the concentration of phase $B$ is low $\left(n_{B}<n_{A}\right)$, because in that case not only the toplayer particles contribute to the intensity, but also deeper lying particles (no 'shadow-effect'). On an increase of $\mu_{\lambda}$, the particles of phase A start to provide 'shadow', resulting in a steep increase of $\mu_{B}^{\text {eff }}$.

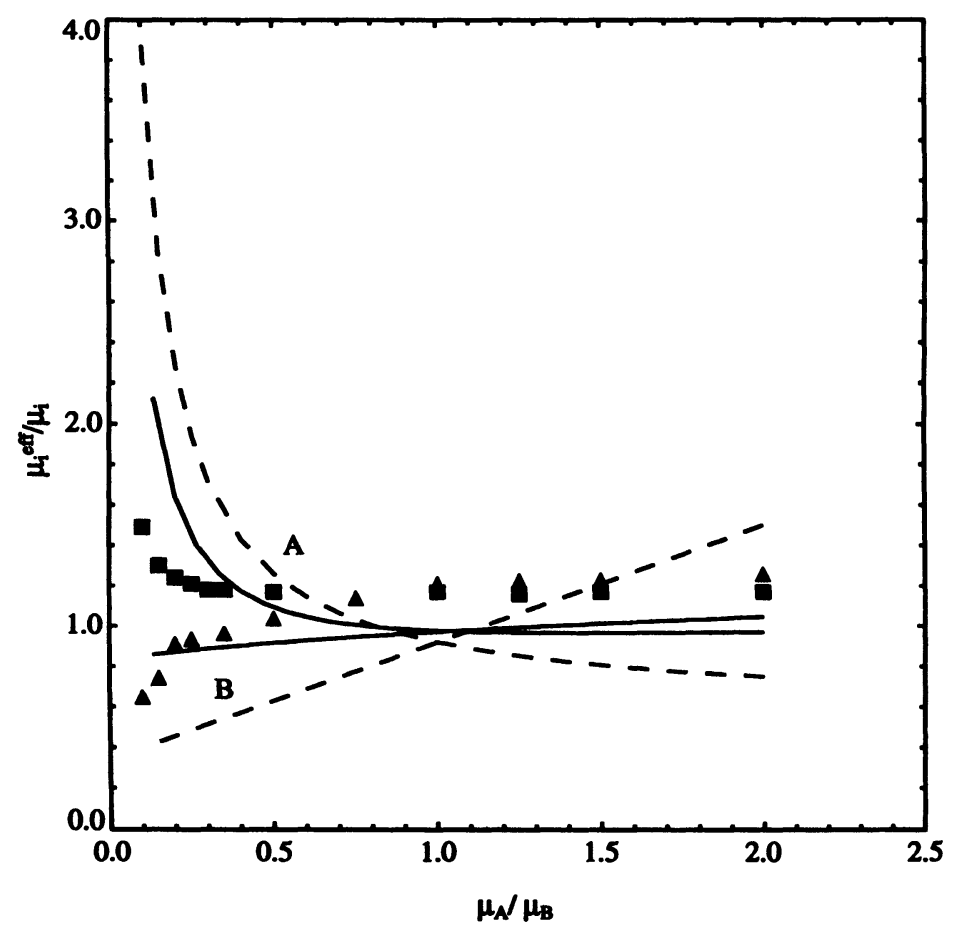

Figure 3 The same as in Figure 2, but with $\mu_{B}=4$. 


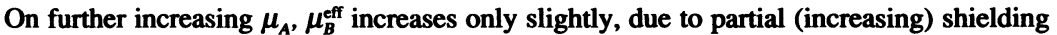
of toplayer particles, until $\mu_{A}$ equals $\mu_{B}$. This 'shielding' effect is caused by the surface roughness. On a further increase of $\mu_{A}, \mu_{B}^{\text {eff }}$ remains constant. The reason that $\mu_{t}^{\text {eff } / \mu_{t}}$ is slightly larger than 1 is also due to surface roughness, which is very important for large $\langle\mu\rangle$ (see below). A complicating problem here is that when the surface roughness contribution becomes of large importance, also particle statistics becomes increasingly more important - thus rendering the statistical description increasingly less valid. As a result, the analytical description of the microabsorption effect is much less succesful in Figure 3 compared to the situation in Figure 2. It may be anticipated, however, that adding effects from surface roughness may improve Eq. (6) (Hermann 1987).

An estimate of the effects of particle statistics can be obtained from Figure 3 (and, to a smaller extent, from Figure 2). For a situation where $\mu_{A}=\mu_{B}$, $\mu_{A}^{\text {eff }}$ should equal $\mu_{B}^{\text {eff }}$. As there is a difference of approximately $5 \%$, it is clear that even for $\sim 2000$ particles, particle statistics can be very important (the more so for larger $\langle\mu\rangle)$.

For practical purposes, it is convenient to know $\mu^{\text {eff }}$ as a function of concentration. Therefore also some calculations have been performed to obtain some feeling for the dependence of $\mu$ on the constituent concentrations, $n_{i}$. In Figures 4 and 5, results from

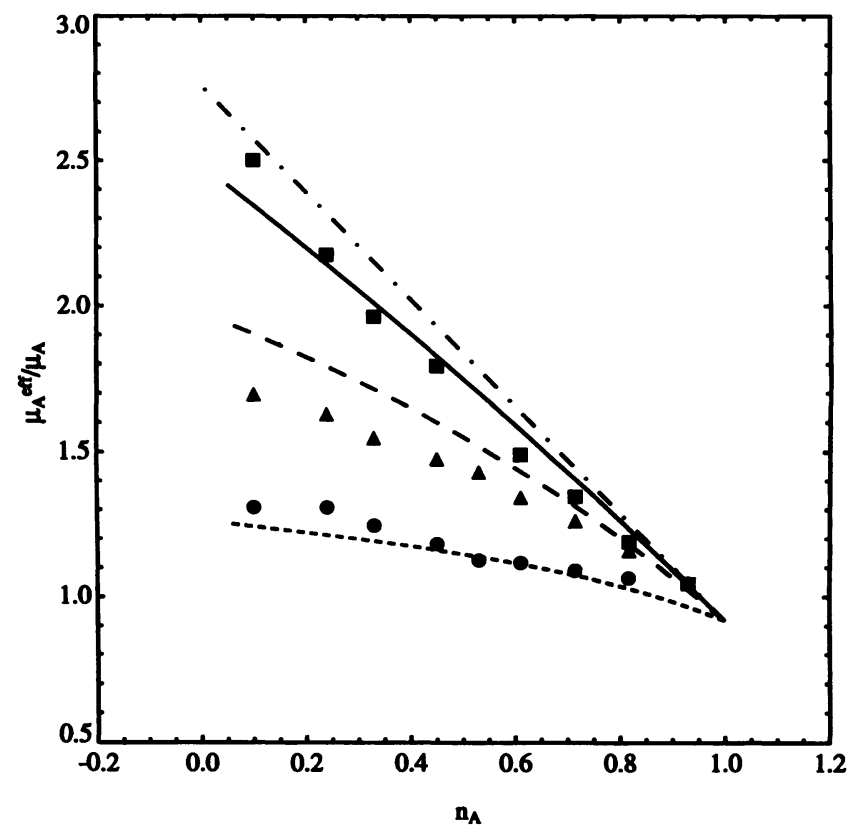

Figure 4 Effective absorption coefficient $\mu_{A}^{\text {eff }} / \mu_{A}$ as a function of the fraction of phases. according to Eq. (6). Drawn: $\mu_{A}=0.08, \mu_{B}=0.24$. Dashed: $\mu_{A}=0.25, \mu_{B}=0.75$. Dotted: $\mu_{A}=1, \mu_{B}=$ 3. The numerical results are represented by squares $\left.\mu_{A}=0.08, \mu_{B}=0.24\right)$, by triangles $\left(\mu_{A}=0.25\right.$, $\left.\mu_{B}=0.75\right)$ and by circles $\left(\mu_{A}=1, \mu_{B}=3\right)$. The dash-dotted line represents Eq. (9). The incidence angle was $\theta=75^{\circ}$ to minimize surface roughness effects. 


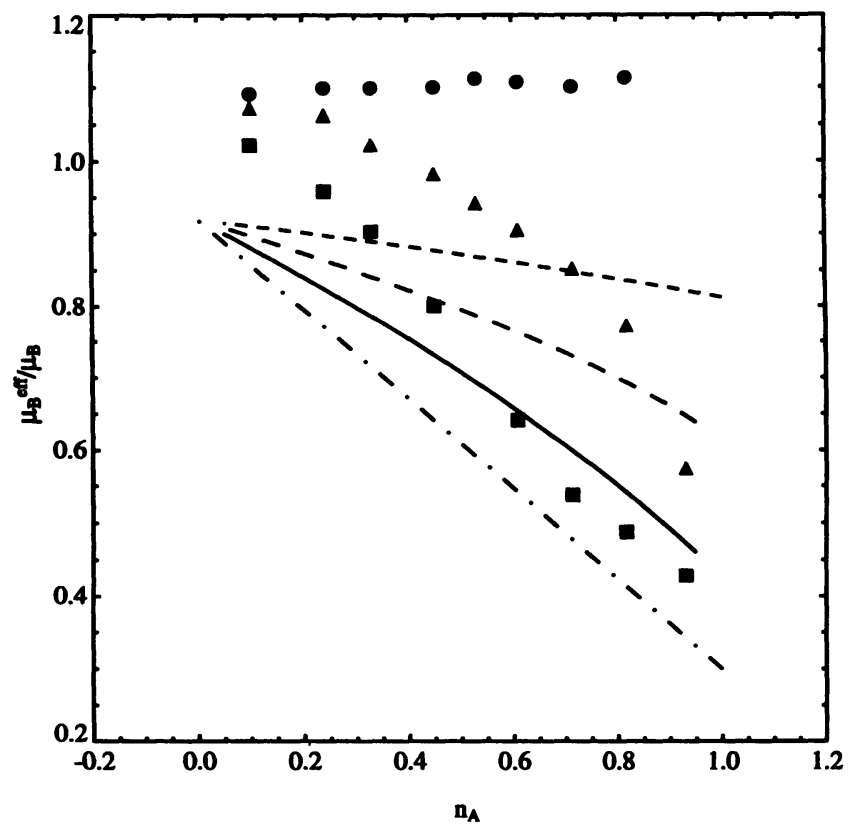

Figure 5 The same as Figure 4 , but for $\mu_{B}^{\text {eff }} / \mu_{B}$.

Eq. (6) are displayed, both for low, medium and high absorption (all with $\mu_{B}=3 \mu_{A}$ ). For low $\mu$, Eq. (9) is approximately followed. For high $\mu$, also the high $\mu$ limit (Eq. (10)) is approximately obeyed. The interesting part of course is the range of intermediate values of $\mu$, where a non-linear behaviour is displayed. In fact, this also is the usual experimental range, where $\langle\mu\rangle \sim 0.1-1$. For the largest $\mu$ values in Figure 5 calculated from Eq. (6), we see that Eq. (10) is obeyed to within $10 \%$ over almost the full concentration regime for $\mu_{B}^{\text {eff }}$, whereas for $\mu_{A}^{\text {eff }}$ (Figure 4) a variation of approximately $30 \%$ is still visible. This indicates, that while for a certain component one of the limiting formulas may hold, it not necessarily holds for other components as well.

As can be seen from Figures 4 and 5, it is not unrealistic to be off by more than a factor of 2 from the real $\mu$ when using Eq. (9), thus causing an error in the intensity by the same factor. It is therefore crucial to be able to give a rather accurate estimate of $\mu$.

To check the assumption on the statistical distribution of particles, the above results are compared with the simulations. In Figures 4 and 5, results from numerical simulations (with incidence angle $\theta=75^{\circ}$ to reduce surface roughness effects) are displayed. In the low $\mu$ region in Figure 4, again the expected linear relationship is obeyed, though still deviations as large as $15 \%$ are visible for $\mu^{\text {eff }} \sim 0.1$. Unfortunately, it is not possible to run the simulations in a reasonable amount of time for still smaller $\mu^{\text {eff }}$, because of convergence problems. 
A striking feature is the rather good correspondance between the results from the simulation and Eq. (6) for $\mu_{A}^{\text {eff }}$ (Figure 4), compared to the large discrepancies for $\mu_{B}^{\text {eff }}$ (Figure 5). This is a result of the surface roughness, which enhances the effect of particle statistics. The values for $\mu_{A}^{\text {eff }}$ are too low (as is most clearly evidenced by the middle curve in Figure 4), whereas the values for $\mu_{B}^{\text {eff }}$ are too high. As the sample surface in the simulations was relatively enriched with phase $A$ at low $n_{A}$ (purely a statistical effect - no phase segregation!), this is an expected effect. In the usual experimental situations, however, with irridiated areas of roughly $1 \mathrm{~cm}^{2}$, this effect will be strongly reduced because of better particle statistics. In experiments using pointfocus and large grains (as e.g. is often the case in metals!) this effect can be as serious as displayed here.

\subsection{Surface roughness}

In the analytical calculations, surface roughness was not taken into account. In the simulations, however, this phenomenon is included quite naturally due to the nature of the model sample. Therefore, in the simulations, some $\theta$ dependence of $\mu^{\boldsymbol{e}^{\text {ff }}}$ should be expected. In Figure 6, results are presented on a case of medium $\langle\mu\rangle$, and of large

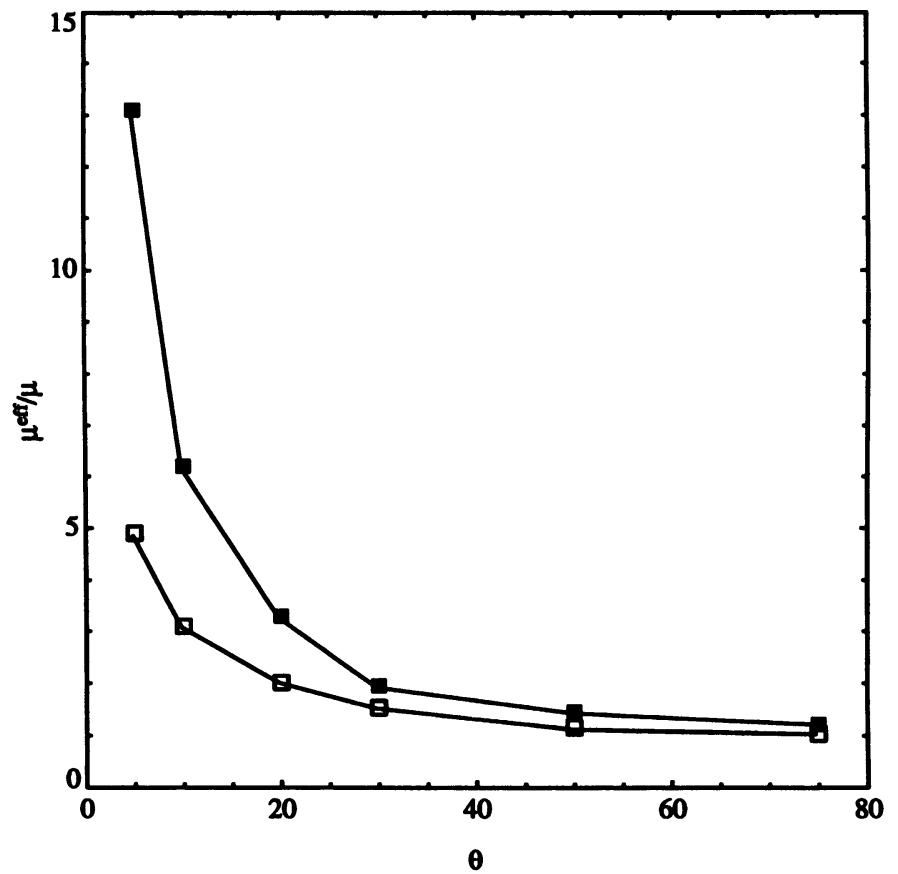

Figure 6 Effective absorption coefficient $\mu^{\text {eff }}$ for a single component sample, as a function of incidence angle $\theta$ (Bragg Brentano geometry). Squares are for $\mu=0.35$, triangles for $\mu=4$. 
$\langle\mu\rangle$, both for a single-component material. The filling fraction for both systems was 0.92; the mean squared surface roughness $\left(\sqrt{\left\langle(h-\langle h\rangle)^{2}\right.}\right)$, with $h$ the local surface height) was approximately $0.5\langle d\rangle$. It is clear, that (certainly for large absorption), surface roughness can be extremely important at low angle of incidence. At low angles, large toplayer grains are highly effective in shadowing smaller or sub-toplayer particles, which therefore do not contribute to the reflected intensity. This effect is becoming less drastic when lowering $\langle\mu\rangle$, as is illustrated in Figure 6.

Another limit, which has not been realized in the simulation, is probably the situation where surface roughness is so strong, that $\mu^{\text {eff }} / \mu$ is decreasing below 1 , simply because the filling fraction is effectively very low in the surface area. In Figure 6, this trend is not visible; even for high angles of incidence, $\mu^{\mathrm{eff}} / \mu$ remains slightly above unity.

\subsection{Nonspherical particles}

To obtain an estimate of the importance of the degree of isotropy of the particles with respect to $\mu^{\text {eff }}$, a few simulations with elongated ellipsoids ('needles') and with flattened ellipsoids (platelets) have been done. In both cases, the ratio of the longest to the shortest axis was 2.5. Moreover, runs with these particles with and without preferred orientation (longest axis preferably parallel to the sample surface) have been performed. Both in the case of needle shaped and platelet shaped particles, a gaussian distribution with a width of $60^{\circ}$ was used to describe the 'texture'. The simulations with the needles were problematic, in that sense, that in the case of needle shaped grains, the maximum filling factor that could be achieved was approximately 0.65 . Only when allowing for an excessive amount of small particles, thus causing a very serious deviation from a gaussian particle size distribution, a higher filling fraction could be reached. This reflects the real-life situation: powders with needle-shaped crystals can not be packed as dense as powders with spherical or platelet shaped particles.

In Figure 7 , the results for $\mu^{\text {eff }}$ as a function of concentration for needle shaped particles are displayed, with and without texture. In the simulation, a rather asymmetric distribution function was used and a filling factor of approximately 0.67 . The average particle size, defined as $\left\langle\sqrt{a^{2}+b^{2}+c^{2}}\right\rangle(a, b, c$ are the lengths of the ellipsoid along its principal axes), is approximately the same as used in the simulations with spherical particles. The presence of texture has little influence on the absorption. This result can be understood from the fact, that the variance in internal distances in a needle in general is not large. Therefore, large effects can only be expected in the improbable situation, when the long axes of the needles are parallel to the incident $\mathrm{X}$-rays.

The results for platelet shaped particles do show some more dependence on texture. In Figure 8, some results for $\mu^{\text {eff }}$ are displayed. The values obtained differ significantly from those for the spherical particles, as can be inferred from direct comparison with Figures 4 and 5; depending on the concentration, discrepancies of roughly $20 \%$ can be observed. Over the full concentration interval $n_{A}=0.25$ to $n_{A}=0.75$, differences in $\mu^{\text {eff }}$ between textured and non-textured samples are approximately $10 \%$, which is a not unreasonable number in view of the previously mentioned effect of $20 \%$. The increase of the effect of the texture with respect to the needle-shaped crystals can be explained on the basis of the increased variance in internal distances in a platelet with respect to a needle. 


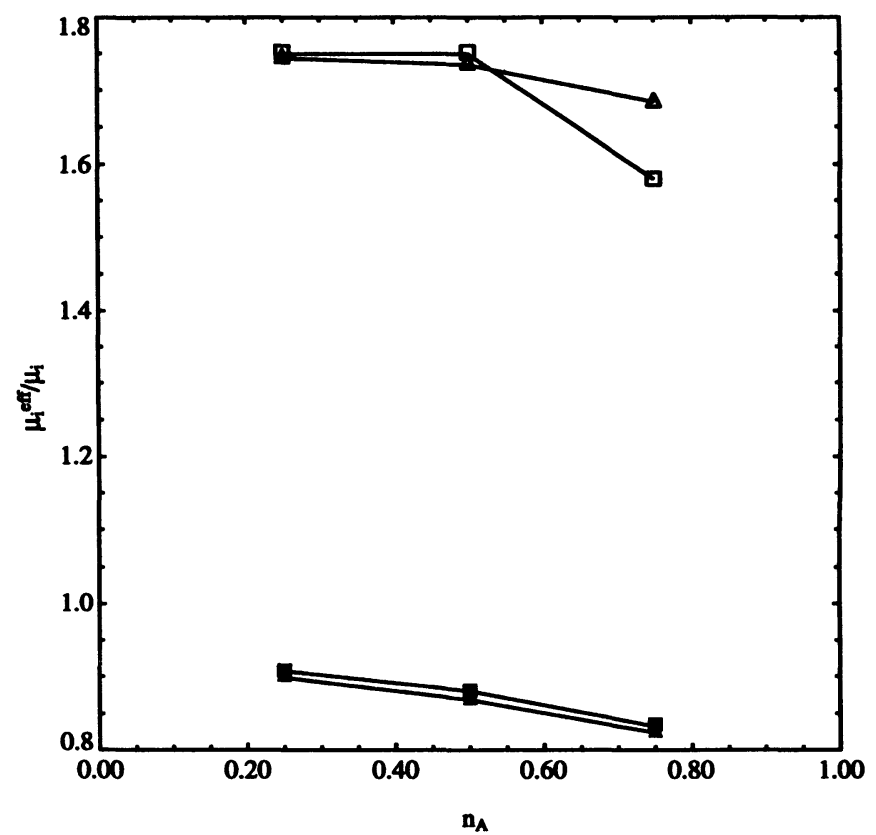

Figure 7 Influence of texture on the effective absorption coefficient for needle-shaped grains. Upper curve: $\mu_{A}^{\text {eff }} / \mu_{\text {; }}$ squares: without texture. Lower curve: $\mu_{B}^{\text {eff }} / \mu_{B}$; curves with and without texture coincide. The filling fraction is $0.67 ; \mu_{A}=0.25 ; \mu_{B}=0.75$.

\section{CONCLUSIONS}

The analytical model (6) gives a much better description of the absorption in non-homogeneous multi-phase powders (in cases were surface roughness effects are not too important) compared to the simple model $(9,10)$, as evidenced by comparison with numerical simulations. Therefore the analytical algorithm is expected to be useful in procedures were correct intensities are important. The limits of the algorithm presented, are in the fact that different average grain diameters and anisotropic shapes of the grains are not allowed for. From the numerical simulations, the error due to the latter imperfection is shown to easily amount to $20 \%$; errors due to preferred orientation can be estimated to be approximately $10 \%$. Though the computer simulation gives more accurate results, especially because surface roughness is included (in contrast with the analytical approach), for routine use only the semi-analytical expressions derived are presently useful in view of the time consuming nature of the simulation. Another approximation is that the kinematical approximation is used throughout the whole calculation. Especially for powders consisting of not too small grains (larger than $5 \mu \mathrm{m})$ of good quality, dynamical effects (e.g. extinction) are expected to become important (an estimation shows that this neglect can easily give errors of $\sim 10 \%$ ). 


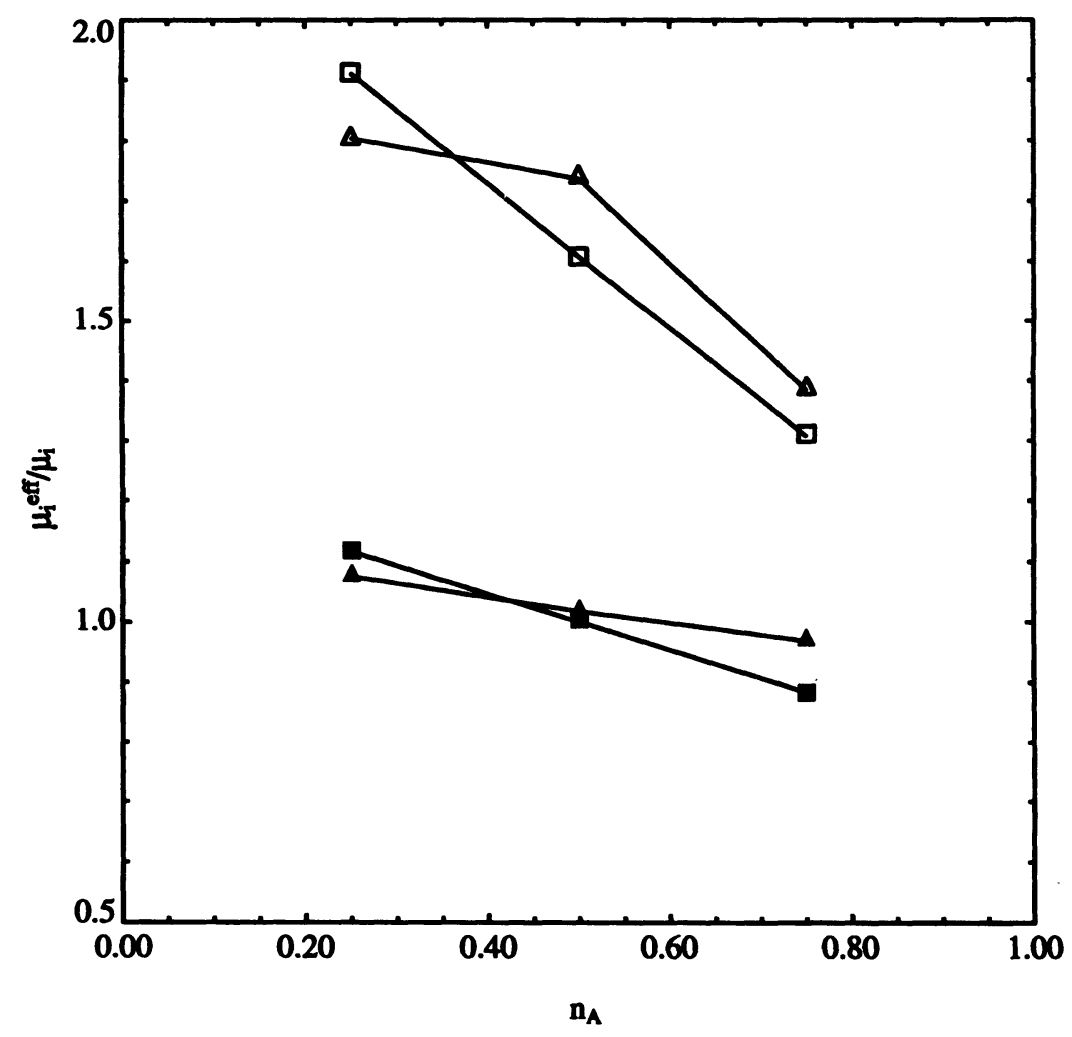

Figure 8 Influence of texture on the effective absorption coefficient for platelet-shaped grains. Upper curve: $\mu_{A}^{\text {eff }} / \mu_{A}$ squares: without texture. Lower curve: $\mu_{B}^{\text {eff }} / \mu_{B}$; squares without texture. The filling fraction is $0.92 ; \mu_{A}=0.25 ; \mu_{B}=0.75$.

Also shown is the drastic effect surface roughness can have on the reflected intensities. In practice, however, this might not be too serious for Bragg-Brentano geometry; the effect is drastic only at low angles of incidence (corresponding to large d-spacings) and for strong absorption. Inorganics usually have not too small d-spacings, and for organics the absorption is in general rather low. For grazing incidence geometries, this might be a serious problem, however; even more serious than the micro absorption effects.

A practical problem which will remain, however, after all above mentioned deficiencies would have been solved, is the problem how to measure correctly all particle size and shape distributions needed for correct evaluation of $\mu^{\text {eff }}$. 


\section{ACKNOWLEDGEMENTS}

The author would like to thank P. Fewster for valuable discussions on dynamic diffraction effects in powders.

\section{References}

Ahtee, M., Nurmela, M., Suortti, P. and Jarvinen, M. (1989). J. Appl. Cryst., 22, 261-268

Brindley, G. W. (1945). Phil. Mag., 36, 347.

Bunge, H. J., and Gao, B. (1992). Proc. 'EPDIC2', The Netherlands, p57.

Hermann, H., and Ermrich, M. (1987). Acta Cryst., A43, 401.

Hermann, H. and Ermrich, M. (1992). Proc. Int. Conf., 'Accuracy in Powder Diffraction II'.

Otto, J. (1984). Z. f. Kristallographie 167, 55.

Reichl, L. E. (1980). in 'A Modern Course in Statistical Physics', Edward Arnold Publishers Ltd. USA.

Suortti, P. (1972). J. Appl. Cryst., 5, 325. 